\title{
Conjugate Immunofluorescence - SEM Array Tomography for Studying Synapses and Other Subcellular Structures in the Brain.
}

\author{
Kristina D. Micheva ${ }^{1}$ \\ 1. Stanford University School of Medicine, Department of Molecular and Cellular Physiology, Stanford, \\ CA, USA
}

Most mammalian synapses are small structures, less than $1 \mu \mathrm{m}$ in diameter, they are tightly packed in the brain and have diverse molecular composition. This makes them a difficult target for studying with a single imaging modality. Electron microscopy is the method of choice for identifying individual synapses and observing their ultrastructure, however it can provide only limited molecular information. Immunofluorescence has the advantage of multiple antibodies use, but cannot reliably distinguish individual synapses within brain tissue. We developed conjugate immunofluorescence - SEM array tomography which allows the imaging of both the molecular content and the ultrastructure of tissues. The method is based on physical ultrathin serial sectioning, immunostaining and acquiring fluorescence and electron microscopy images of resin embedded tissues, followed by computational volume reconstruction and analysis.

Tissue preparation and imaging. Adult mice were perfusion fixed with $2 \%$ glutaraldehyde and $2 \%$ formaldehyde in phosphate buffer. Vibratome sections $(200 \mu \mathrm{m})$ were cryoprotected with glycerol, freeze-substituted and embedded in Lowicryl HM-20 [1]. Serial $70 \mathrm{~nm}$ sections were collected on carbon-coated glass coverslips. Sections were processed for standard indirect immunofluorescence and imaged on an automated epi-fluorescent microscope (Zeiss AxioImager Z1) using a 63x Plan Apochromat 1.4 N.A. oil objective [2]. After immunofluorescence imaging, ribbons were poststained with uranyl acetate and lead citrate and imaged on a Zeiss Sigma Field Emission SEM using the backscatter detector. Image registration (between different fluorescence imaging sessions, and between fluorescent and electron microscopy mages) and alignment (within the stack of serial images) was done using the open source software FIJI. Fluorescence intensity measurements were performed using FIJI.

Results. Osmium-free freeze-substitution embedding in Lowicryl HM-20 provides excellent ultrastructure, allowing the identification of synapses based on the presence of synaptic vesicles and postsynaptic densities. The majority of antibodies previously validated on formaldehyde-fixed, LR White embedded tissue, can be successfully used. Furthermore, additional antibodies, for example against small molecules (GABA), requiring the presence of glutaraldehyde in the fixative, can also be applied.

Summary. Cryoembedding in Lowicryl HM-20 preserves both antigenicity and ultrastructure of brain tissue, enabling conjugate array tomography. Using fluorescence microscopy, dozens of different antibodies and other fluorescent markers can be imaged at synaptic resolution within large volumes of tissue. The same arrays can also be viewed using field emission SEM, which allows for the classic benefits of electron microscopy. Such integration of different imaging modalities can substantially enrich our understanding of the brain. 

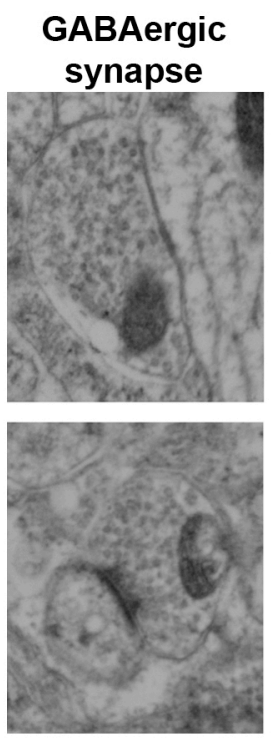

Glutamatergic synapse
GABA + Gephyrin + VGluT1 + PSD95
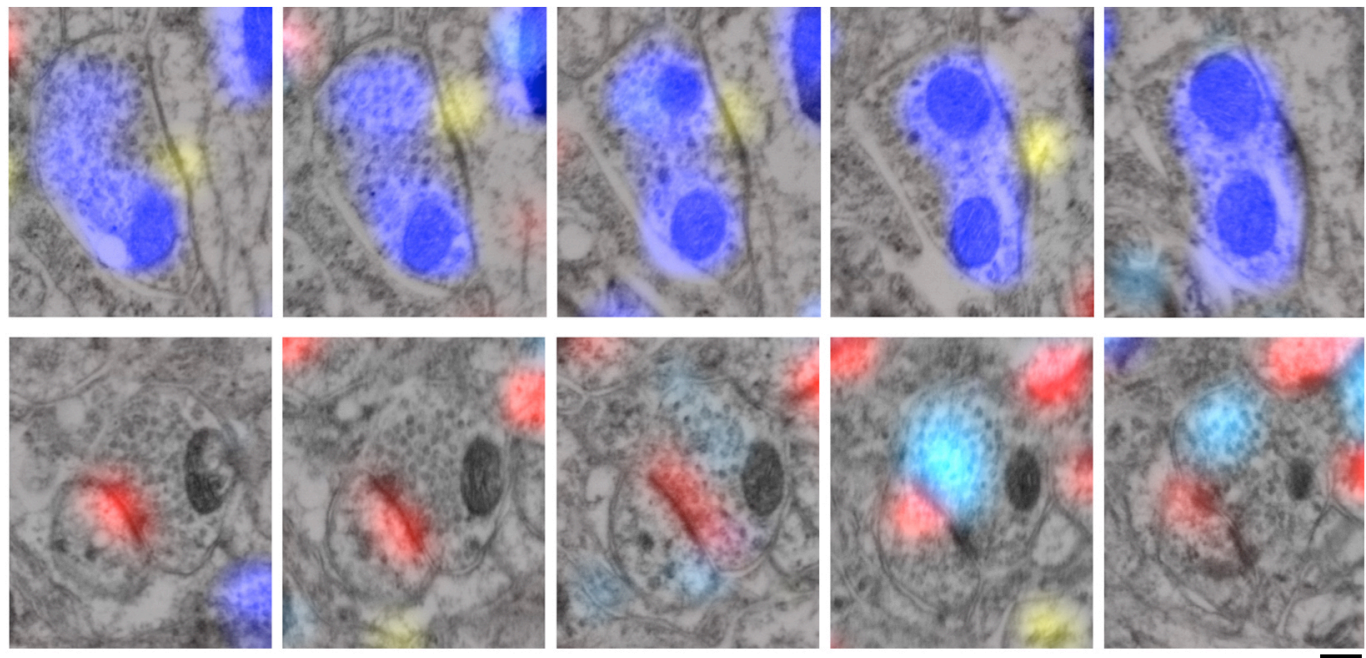

Serial sections

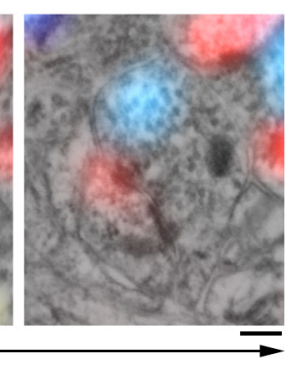

Figure 1. Immunofluorescence and ultrastructure of a GABAergic and glutamatergic synapse in adult mouse neocortex. GABA, blue, gephyrin, yellow, VGlut1, cyan and PSD95, red. Scale bar, $0.2 \mu \mathrm{m}$.

\section{GABA + MBP + $\alpha$ Tubulin + DAPI}
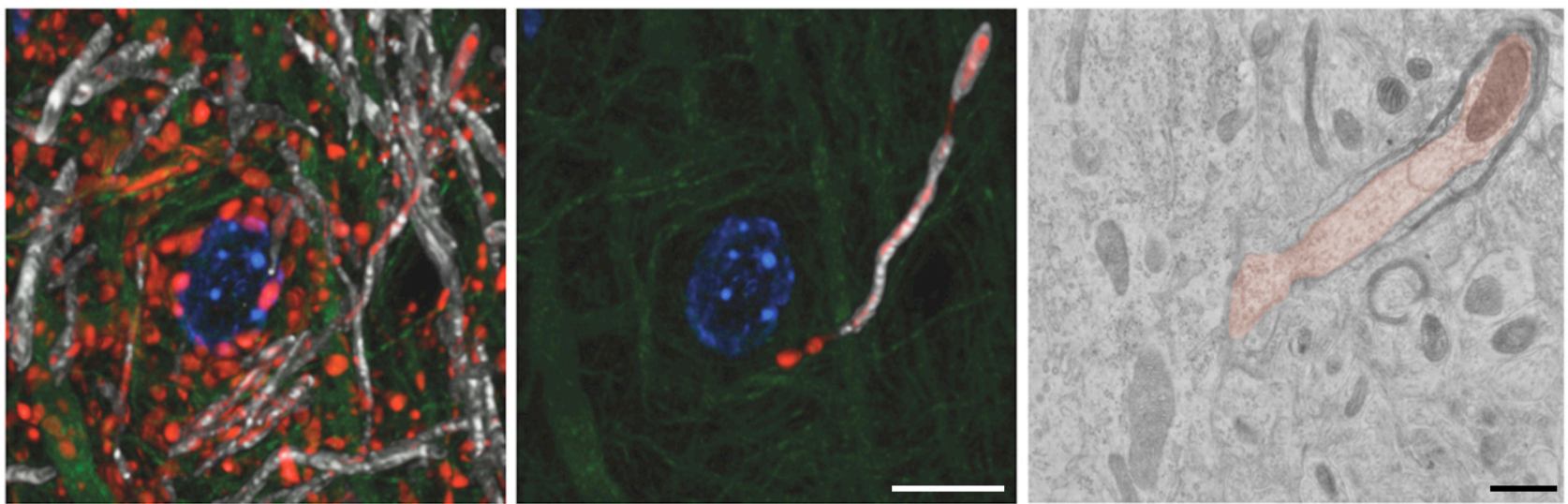

Figure 2. A myelinated GABAergic axon making a synaptic contact on a neuronal cell body in the adult mouse neocortex. The two images on the left are volume reconstructions from 40 serial sections, $70 \mathrm{~nm}$ each. GABA, red, myelin basic protein (MBP), white, $\alpha$ tubulin, green, and DAPI, blue. Scale bar, $5 \mu \mathrm{m}$. The SEM image to the right shows the same axon making a synaptic contact on the cell body. GABA, red. Scale bar, $0.5 \mu \mathrm{m}$.

References:

[1] JD Ding, MB Kennedy and RJ Weinberg, J. Comp. Neurol. 521 (2013), p.3570.

[2] KD Micheva, B Busse, NC Weiler, N O'Rourke and SJ Smith, Neuron 68 (2010), p.639.

[3] The author wishes to thank Stephen J Smith, Richard J. Weinberg, JoAnn Buchanan and Forrest Collman for their invaluable scientific input and continuing support, and Kristen Phend and Nafisa Ghori for tissue preparation. This work was supported by grants from the National Institutes of Health (R21MH099797, R01NS075252, R01NS077601 to SJS, R01NS039444 to RJW). 